\title{
Polarization, Barriers and Tunneling Times of Some Tautomers of Adenine and Thymine
}

\author{
Julio Marañon* and Oscar M. Sorarrain** \\ Departamento de Fisica, Facultad de Ciencias Exactas, Universidad Nacional de La Plata. \\ 1900 La Plata, Republica Argentina
}

Z. Naturforsch. 34a, 315-319 (1979); received December 22, 1978

\begin{abstract}
The polarization of the radiation involved in some transitions is analysed for the normal and tautomeric forms of adenine and thymine. Tunneling times for some barriers previously reported [1] and equilibrium constants between two isomers for some excited states are also given. The calculations have been made with the CNDO/2-CI method and the results compared with the available experimental data.
\end{abstract}

\section{Introduction}

The relative stability of the normal and tautomeric forms of adenine and thymine has been formerly analysed with semi-empirical and abinitio methods $[1-2]$. In the same way we have now calculated the charge distribution of several excited states in order to determine the intramolecular charge transfer involved in the tautomerization process. Moreover, we have obtained the direction of the polarization of the light emitted during transitions between some excited states and the ground state. From the barriers reported in a preceding paper [1] some characteristic parameters for the tunneling and the equilibria between the tautomers of adenine and thymine were obtained.

\section{Method and Results}

\section{a) Radiation Polarization and Dipole Moment}

Pullman [2] has given the direction of the polarization of the lines due to transitions between the ground and several excited states of the nucleotide bases in their normal forms. In this paper, we have extended the calculations to the tautomeric forms of adenine and thymine using a CNDO/2 standard program.

Adenine has several singlet states in the first and third region of the spectra classification given by Clark [3]. The calculated values for these states are given in Table 1.

* Miembro Carrera del Investigador, CONICET.

** Miembro Comision de Investigaciones Cientificas, Pcia de Bs. As.

Reprint requests to Dr. O. M. Sorarrain. Please order a reprint rather than making your own copy.

$0340-4811 / 79 / 0300-0315 \$ 01.00 / 0$
Table 1. Transition lines for adenine given in Fig. 1, energies and oscillator strengths, a) first, b) third spectral region. Energies in eV, oscillator strengths in brackets.

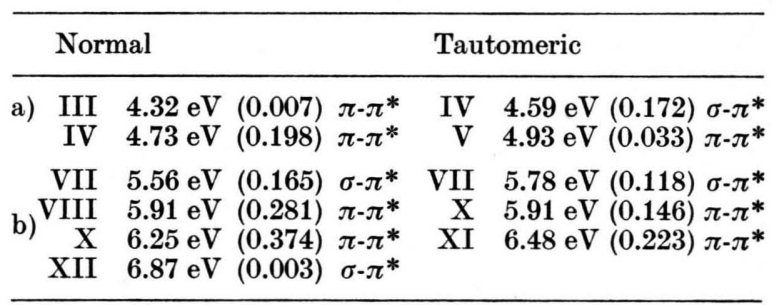

The directions of the polarization of the radiation involved in the transitions between these states and the ground state are given in Figure 1. In this figure, the dipole moment of the ground state is given in a coordinate system with its origin on the baricenter of the molecule. Our calculations should be compared with the experimental data reported by Mason [4]. This author has experimentally analysed the polarization of the corresponding lines of 9-methyladenine. He has concluded that there is a strong longitudinal component and a weak transversal one in the $\mathrm{C}_{4}-\mathrm{C}_{5}$ direction, see Figure 1 . In our calculations for the normal form the transition corresponding to the III state has a $30^{\circ}$ angle with the $\mathrm{C}_{4}-\mathrm{C}_{5}$ direction, and the transition line of the IV state is almost in the longitudinal direction.

For the third spectral region our calculations predict mainly longitudinal polarizations for the transition lines corresponding to the X and XII excited states and mainly transversal polarizations for the VII and VIII ones. The agreement between our calculations and the experimental results is in the whole satisfactory for normal adenine, especially if we consider the rather qualitative character of Mason's conclusions. 

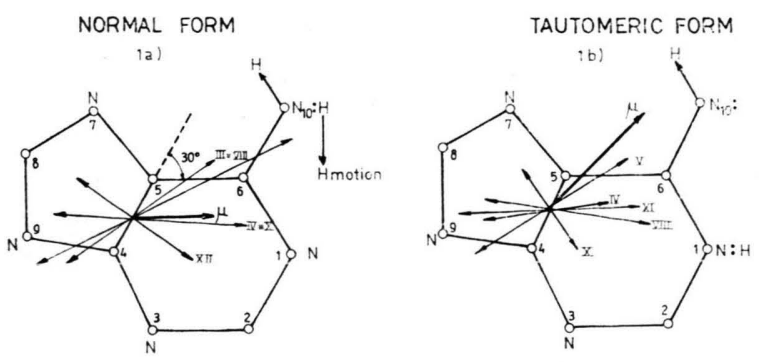

DIPOLE MOMENT

$\longrightarrow \mu=2.7096 \mathrm{Dy}$

DIPOLE MOMENT

$\longrightarrow=4.3003 \mathrm{Dy}$

Fig. 1. Polarization for the transitions given in Table 1, and dipole moment for the ground electronic state of adenine.

Since for the tautomeric form of adenine there are no experimental data available, we only point out that the stronger components involved in the attained transitions are mainly polarized in the transverse direction, see Figure $1 \mathrm{~b}$ ).

The dipole moment of the ground state in the normal form is oriented close to the longitudinal direction of the lone pair orbital $n(\pi)(-9.52 \mathrm{eV})$ belonging to the $\mathrm{N}_{1}$ atom. On the contrary, the transverse dipole moment corresponding to the tautomeric form is due to the lone pair $n(\sigma)$ $(-10.14 \mathrm{eV})$ centered on the $\mathrm{N}_{10}$, see Figure $\left.1 \mathrm{a}\right)$ and $b)$.

For thymine in the spectral regions defined by Clark (3) we have obtained the lines reported in Table 2. The directions of the polarizations corresponding to these transitions and the dipole moments for the ground state of both tautomeric forms are given in Figure 2.

Our calculations for thymine in its normal form can be compared with the experimental results given by Stewart $[5,6]$ in 1-methylthymine. According to this author, the radiation corresponding

Table 2. Transition lines for thymine given in Fig. 2, energies and oscillator strengths, a) first, b) second, c) third spectral region. Energies in $\mathrm{eV}$, oscillator strengths in brackets.

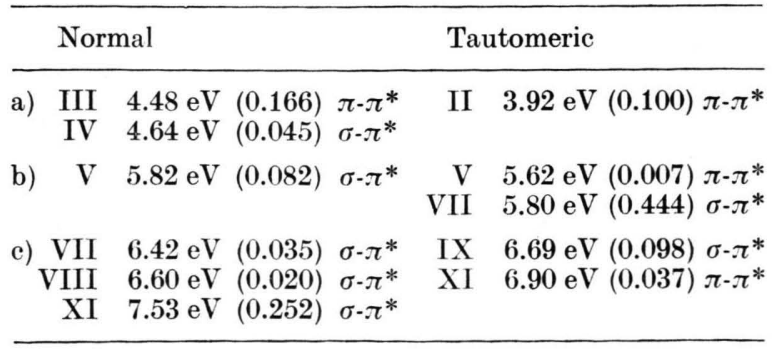
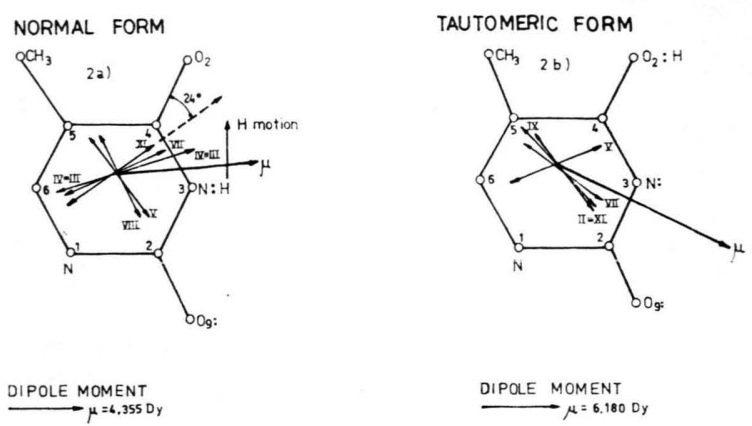

Fig. 2. Polarization for the transitions given in Table 2, and dipole moment for the ground electronic state of thymine.

to the first spectral region has an angle of $19^{\circ}$ with the $\mathrm{N}_{1}-\mathrm{C}_{4}$ direction, and in the second spectral region the direction of polarization is perpendicular to the first one. In this paper, we predict polarizations with $\cong 24^{\circ}$ between the directions corresponding to the first and third spectral regions and the $\mathrm{N}_{1}-\mathrm{C}_{4}$ direction (see XI, VII and IV, Figure 2a)). For the second region, the polarizations are $\cong 90^{\circ}$ to the former ones (see, Figure 2a)). Thus, the experimental data and the theoretical values are in full agreement. For the tautomeric form the direction of the polarizations involved in the excited states reported here are $\cong 90^{\circ}$ with the $\mathrm{N}_{1}-\mathrm{C}_{4}$ direction (see Figure $2 \mathrm{~b}$ )). There is only one exception, the $\mathrm{V}$ transition.

The dipole moment for the normal form of thymine is almost in the $\mathrm{C}_{6}-\mathrm{N}_{3}$ direction. The lone pair orbitals $n(\sigma)(-10.74 \mathrm{eV})$ and $n(\sigma)(-11.65 \mathrm{eV})$ located on the atoms $\mathrm{O}_{8}$ and $\mathrm{O}_{9}$ are the main contributors to the dipole moment. These results are in agreement with former experimental ones obtained by Jordan [7] for dihydrothymine. In a similar way the dipole moment for tautomeric thymine arise from the lone pair orbitals $n(\sigma)(-10.99 \mathrm{eV})$ and $n(\sigma)(-9.99 \mathrm{eV})$ centered on the $\mathrm{N}_{3}$ and $\mathrm{N}_{4}$ atoms.

\section{b) Charge Distributions as a Function of the H Motion}

The variation of the electronic charges of the $\mathrm{H}$, $\mathrm{C}_{6}, \mathrm{C}_{2}, \mathrm{~N}_{1}$ and $\mathrm{N}_{10}$ atoms for adenine in the ground state has been obtained as a function of the $\mathrm{H}$ motion between the normal and tautomeric forms. The results are given in Figure 3.

We can conclude from these calculations that the electronic charges of the atoms $\mathrm{C}_{6}$ and $\mathrm{C}_{9}$ hardly 


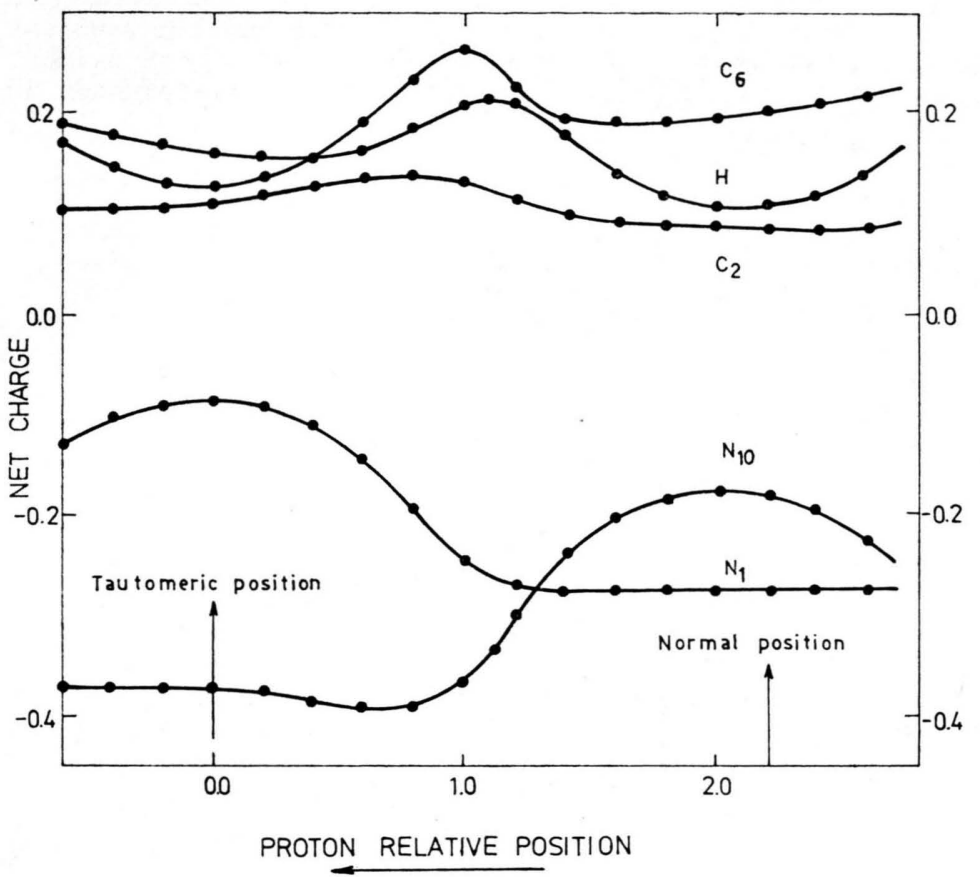

Fig. 3. Charge distributions in course of the $\mathrm{H}$ motion between the normal and tautomeric positions of adenine.

change; on the contrary, large variations are observed for $\mathrm{N}_{1}(\Delta q=-0.17)$ and $\mathrm{N}_{10}(\Delta q=0.19)$, that is practically an intramolecular charge transfer occurs between these atoms. The results obtained in our calculations for the tautomerization process are in agreement with former ones of several authors [8-13]. These authors have concluded that the $\mathrm{N}_{1}$ atom is the most reactive center for a proton attack. Moreover, although the numerical values are not given in this paper, the intramolecular charge transfers mentioned above are also observed for the excited states whose radiation polarizations are given in Figure 1.

A quite similar calculation has been performed for the electronic charges of the $\mathrm{H}, \mathrm{C}_{4}, \mathrm{~N}_{3}$ and $\mathrm{O}_{4}$ atoms corresponding to the ground state of thymine. The results are shown in Figure 4.

We can see in this figure a rather similar intramolecular charge transfer as in the case of adenine, thus for $\mathrm{N}_{3}(\Delta q=0.195)$ and for $\mathrm{O}_{8}(\Delta q=-0.245)$. These values are qualitatively in agreement with those formerly attained by Jordan [8] and Bonaccorsi [10]. The last author concluded that $\mathrm{O}_{8}$ and $\mathrm{O}_{9}$ are the centers for a proton attack. As we have formerly reported for adenine, there is also an intramolecular charge transfer between the atoms $\mathrm{N}_{3}$ and $\mathrm{O}_{8}$ in thymine in the normal-tautomeric process corresponding to the excited states given in Figure 2 .

\section{c) Tunneling and Equilibria}

In a previous paper [1] we have reported the correlation between the normal and tautomeric forms for several excited singlet and triplet levels of adenine and thymine. Moreover, we have determined the shape of the barriers between the configurations shown in Fig. 1 and 2, for the same motion as analysed in this paper.

The quantum-mechanical background for the calculation of the tunneling and equilibria between different tautomers has been summarized by Löwdin [14] and Rein [15]. In this work, we are following their line calculating some characteristic parameters of the tunneling process in the ground and in the excited states given formerly in reference 1.

The probability of an incident particule tunneling through a barrier depends in the WKB approximation on the quantity $s$, given by

$$
s=(2 \pi / h) \int_{x_{1}}^{x_{2}}\left\{2 m[V(x)-E]^{1 / 2}\right\} \mathrm{d} x
$$

where $m$ and $E$ are the mass and energy of the proton, $V(x)$ is the potential energy at position $x$, and $x_{1}$ and $x_{2}$ are the turning points (the limits of the classically forbidden portion of the path

$$
\left.\left(V(x) \geqq E, x_{1} \leqq x \leqq x_{2}\right)\right) .
$$




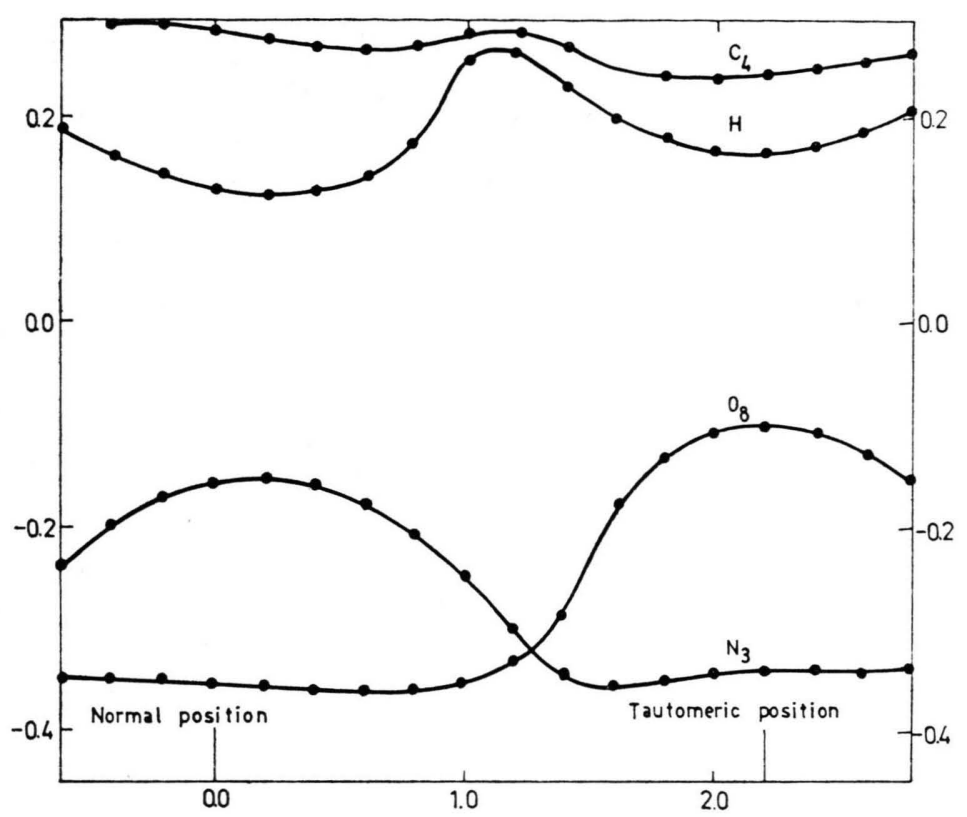

Fig. 4. Charge distribution in course of the $\mathrm{H}$ motion between the normal and tautomeric positions in the case of thymine.

PROTON RELATIVE POSITION

For a parabolic barrier of height $V_{0}$ and width $a_{0}$, the expression for $s$ becomes

$$
s=\left(\pi a_{0} / 2 h\right)\left(2 m V_{0}\right)^{1 / 2},
$$

and the probability $g$ that the incident particle will penetrate the barrier at the minimum tunneling energy is given by

$$
g=e^{-2 \mathrm{~s}} \text {. }
$$

Thus, if the proton is incident upon the barrier $v$ times per second, the tunneling rate, per second (frequency of penetration per particule at the minimum tunneling energy) will be

$$
c=g v .
$$

For the calculation of $v$ it was found more convenient to estimate $v$ directly from the curvature of the minima, assuming them to be parabolic with a potential $V(x)=(\alpha / 2)\left(x-x_{0}\right)^{2}\left(x_{0}\right.$ is the position of the minimum of the potential). For the frequency $v$, we obtained from the theory of the harmonic oscillator

$$
\boldsymbol{v}=(1 / 2 \pi)(\alpha / m) .
$$

For an isolated level, the deviation from the equilibrium (between normal and tautomeric configurations) distribution decays exponentially, that is as $\exp \left(-\left(C_{\mathrm{N}}+C_{\mathrm{T}}\right) t\right)$, so that (see Löwdin [14])

$$
\tau=\left(C_{\mathrm{N}}+C_{\mathrm{T}}\right)^{-1}
$$

can be identified as a characteristic tunneling time (the time needed for a departure from tunneling equilibrium to be reduced by $1 / e$ of its original value). Assuming the minima to be nearly parabolic, the equilibrium proton distribution among the normal and tautomeric forms can be estimated from the expression for the harmonic oscillator partition function in the following way:

$$
\begin{aligned}
K= & \frac{n_{\mathrm{T}}}{n_{\mathrm{N}}}=\exp \left(\frac{-\Delta E}{k T}\right) \frac{\exp \left(-h \nu_{\mathrm{T}} / 2 k T\right)}{\exp \left(-h \nu_{\mathrm{N}} / 2 k T\right)} \\
& \cdot \frac{\left[1-\exp \left(-h \nu_{\mathrm{N}} / k T\right]\right.}{\left[1-\exp \left(-h \nu_{\mathrm{T}} / k T\right]\right.}
\end{aligned}
$$

$\nu_{\mathrm{N}}=$ oscillator frequency, normal form,

$v_{\mathrm{T}}=$ oscillator frequency, tautomeric form;

where $\Delta E$ is the difference between the minimum energies corresponding to the normal and tautomeric forms. The numerical values for $v, g, v_{\mathrm{N}}, v_{\mathrm{T}}$, $\tau$ and $K$ are shown in Table 3 .

We can see from the values for $K$ in Table 3 that tunneling is really negligible in the ground state of both molecules, but its probability is large for some excited states. In this way, radiation can induce 
Table 3. Tunneling time and equilibrium constants for several excited states of adenine and thymine, (s) singlets (t) triplets.

Adenine

\begin{tabular}{|c|c|c|c|c|c|c|}
\hline & $s[1]$ & $g[2]$ & $\nu_{\mathrm{T}}\left(\mathrm{sec}^{-1}\right)[3]$ & $v_{\mathrm{N}}\left(\sec ^{-1}\right)$ & $\tau$ (sec) $[4]$ & $K=n_{\mathrm{t}} / n N$ \\
\hline G.S. & 14.49 & $2.59 \times 10^{-13}$ & $4.87 \times 10^{12}$ & $1.75 \times 10^{12}$ & $5.83 \times 10^{-1}$ & $1.90 \times 10^{-30}$ \\
\hline $1 \sigma-\pi^{*}(\mathrm{~s})$ & 3.90 & $4.10 \times 10^{-4}$ & $4.38 \times 10^{11}$ & $4.38 \times 10^{11}$ & $1.59 \times 10^{-8}$ & $1.04 \times 10^{6}$ \\
\hline $2 \sigma-\pi^{*}(\mathrm{~s})$ & 2.76 & $4.01 \times 10^{-3}$ & $3.46 \times 10^{11}$ & $3.46 \times 10^{11}$ & $3.60 \times 10^{-10}$ & $8.62 \times 10^{-5}$ \\
\hline $3 \pi \rightarrow \pi^{*}(\mathrm{t})$ & 9.78 & $5.20 \times 10^{-9}$ & $6.64 \times 10^{11}$ & $3.88 \times 10^{11}$ & $2.97 \times 10^{-4}$ & $5.84 \times 10^{-1}$ \\
\hline $3 \sigma \rightarrow \pi^{*}(\mathrm{t})$ & 1.55 & $4.50 \times 10^{-2}$ & $7.79 \times 10^{11}$ & $3.46 \times 10^{11}$ & $1.97 \times 10^{-11}$ & $7.84 \times 10^{2}$ \\
\hline
\end{tabular}

Thymine

\begin{tabular}{lllllll}
\hline & $s$ & $g$ & $v_{\mathrm{T}}\left(\mathrm{cm}^{-1}\right)$ & $v_{\mathrm{N}}\left(\mathrm{cm}^{-1}\right)$ & $\tau(\mathrm{cm})$ & $K=n_{\mathrm{t}} / n N$ \\
\hline G.S. & 38.68 & $2.52 \times 10^{-34}$ & $4.49 \times 10^{12}$ & $7.79 \times 10^{13}$ & $4.82 \times 10^{19}$ & $5.69 \times 10^{-24}$ \\
$1 \sigma-\pi^{*}(\mathrm{~s})$ & 8.37 & $5.37 \times 10^{-8}$ & $7.79 \times 10^{13}$ & $3.46 \times 10^{13}$ & $1.66 \times 10^{-7}$ & $4.36 \times 10^{-6}$ \\
$3 \sigma-\pi^{*}(\mathrm{~s})$ & 1.64 & $3.76 \times 10^{-2}$ & $1.75 \times 10^{13}$ & $7.79 \times 10^{13}$ & $2.79 \times 10^{-3}$ & $1.54 \times 10^{10}$ \\
$2 \pi \rightarrow \pi^{*}(\mathrm{t})$ & 8.63 & $3.19 \times 10^{-8}$ & $2.80 \times 10^{13}$ & $5.72 \times 10^{13}$ & $3.68 \times 10^{-7}$ & $2.65 \times 10^{3}$ \\
$1 \sigma-\pi^{*}(\mathrm{t})$ & 8.29 & $6.36 \times 10^{-8}$ & $4.85 \times 10^{13}$ & $1.39 \times 10^{12}$ & $3.15 \times 10^{-7}$ & $1.78 \times 10^{2}$ \\
\hline
\end{tabular}

tautomerization by a previous transition between the ground state and the corresponding excited levels.

\section{Conclusions}

The preceding paragraphs could be summarized in the following way:

a) For the transitions considered in this paper the polarization of the stronger lines is nearly parallel to the dipole moment of the ground state in the normal and tautomeric forms of both molecules.

b) The lone pair orbitals strongly influence the direction of the dipole moment in the ground

[1] J. Marañon and O. M. Sorarrain, Z. Naturforsch. 32 e, 647 (1977).

[2] A. Pullman and B. Pullman, Advances in Quantum Chemistry, Vol. 4, Academic Press, New York 1968, p. 267.

[3] L. B. Clark and I. Tinoco, Jr., J. Amer. Chem. Soc. 87, 11 (1965).

[4] S. F. Mason, J. Chem. Soc. 1954, 2071.

[5] R. F. Stewart and N. J. Davidson, J. Chem. Phys. 39, 255 (1963).

[6] R. F. Stewart and L. H. Jensen, J. Chem. Phys. 40, 2071 (1964).

[7] F. Jordan, Theor. Chim. Acta 11, 390 (1968).

[8] F. Jordan and H. D. Sostman, J. Amer. Chem. Soc. 95, 6544 (1973). states of both tautomeric forms of both molecules.

c) The tunneling effect through the barrier reported in our previous work [1] is negligible in the ground state but large enough to be considered in some excited states in both molecules.

\section{Acknowledgements}

We should like to express our gratitude to the Consejo Nacional de Investigaciones Cientificas de Argentina and to the Organization of the American States for their support of this research.

O. M. Sorarrain is also grateful to the Comision de Investigaciones Cientificas, Pcia de Bs.As. for the personal grant received from this Institution.

[9] E. Scrocco and J. Tomassi, J. Chem. Phys. 52, 5270 (1970).

[10] R. Bonaccorsi, A. Pullman, E. Scrocco, and J. Tomassi, Theor. Chim. Acta 24, 51 (1972).

[11] C. Giessner-Prette and A. Pullman, Theor. Chim. Acta 25, 83 (1972).

[12] C. Giessner-Prette and A. Pullman, Theor. Chim Acta 33, 91 (1974).

[13] R. Bonaccorsi, E. Scrocco, J. Tomassi, and A. Pullman, Theor. Chim. Acta 36, 339 (1975).

[14] P. O. Löwdin, Quantum Genetics and the Aperiodic Solid, Preprint QB 7, Quantum Chemistry Group, Uppsala University, 1963.

[15] R. Rein and F. E. Harris, J. Chem. Phys. 42, 2177 (1965). 